\title{
Gestão internacional comparativa de recurso humanos e gestão de recursos humanos no Brasil: Uma análise frente aos modelos calculativos e colaborativos
}

\author{
Tatiani dos Santos Zuppani ${ }^{1}$ e André Luiz Fischer \\ Universidade de São Paulo - USP, São Paulo, SP, Brasil.
}

DETALHES DO ARTIGO
Histórico do artigo:
Recebido em 11 de janeiro de 2016
Aceito em 28 de abril de 2016
Disponível online em 31 de agosto de 2016
Sistema de Revisão "Double Blind Review"
Editor científico:
Eduardo Eugênio Spers

\section{Palavras-chaves:}

Gestão Internacional Comparativa de

Recursos Humanos;

Gestão Estratégica de Recursos Humanos;

Modelo Calculativo;

Modelo Colaborativo

\begin{abstract}
RESUMO
O objetivo deste estudo foi analisar a adoção de práticas calculativas e colaborativas, dominantes na gestão internacional comparativa de recursos humanos, segundo os diferentes perfis das áreas de Gestão de Recursos Humanos (GRH) de organizações privadas atuantes no Brasil. O método empregado foi um Survey, operacionalizado por meio de um questionário eletrônico sobre práticas de GRH e características organizacionais. Foram obtidos um total de 326 respondentes. Inicialmente foi realizado um cluster no qual os respondentes foram agrupados em quatro grupos com perfis de GRH distintos. A utilização de práticas calculativas e colaborativas foi comparada nos quatro grupos formados através de um teste Anova. Os principais achados mostraram que o grupo estratégico foi aquele com maior média de adoção de práticas calculativas e colaborativas. O grupo GRH Comunicativa apresentava uma propensão maior para práticas colaborativas e o grupo GRH Formalizada adotava práticas calculativas, embora nenhum dos grupos apresentou uma média de adoção do que o grupo GRH Estratégico. Isto sugere que é preciso aprender a lidar com aspectos diferentes que envolvem a gestão de pessoas nas organizações que atuam no Brasil.
\end{abstract}

(C) 2016 Internext | ESPM. Todos os direitos reservados!

\section{Introdução}

O gerenciamento dos empregados é fortemente influenciado por fatores contextuais e culturais do cenário no qual a organização se insere (Croucher, Gooderham, \& Parry, 2006; Tanure, Evans, \& Cançado, 2010a). Essa afirmação torna coerente a evolução da área de Gestão de Recursos Humanos (GRH) de uma atuação operacional para uma postura mais estratégica. Dessa perspectiva, diferentes variáveis precisam ser consideradas, inclusive aquelas relacionadas ao contexto no qual as organizações se inserem. A compreensão de aspectos macroambientais, portanto, tem ganhado espaço e desencadeado estudos de GRH contextualizados (Brewster, 2004; Kramar, 2012; Peng, 2005), especialmente frente à competitividade global das organizações e à evolução da Gestão Internacional de Recursos Humanos (GIRH).

As organizações que atuam no Brasil sofrem influência do ambiente externo à elas. A partir dos anos 1990, com a abertura econômica nacional, ocorreram grandes reformas estruturais, acompanhadas pelo aumento do número de expatriados, transmissão de novas formas de gestão difundidas pela mídia empresarial, por consultorias e escolas de negócio (Chu \& Wood Jr., 2008) e aumento da influência de diferentes organizações multinacionais. Além disso, a tecnologia promove o encurtamento das distâncias, e em conjunto com outros fatores alavanca a globalização que acirra a disputa entre as organizações, agora com concorrentes espalhados pelo mundo, tornando a 
eficiência um imperativo de sobrevivência, que depende dos resultados produzidos pelos empregados das organizações e da capacidade deles em responder às demandas ambientais.

Essa conjuntura tem levado à $\mathrm{GRH}$ das organizações que atuam no Brasil a mudar, visto que elas precisam se adaptar a essas demandas contextuais. Esse aspecto potencializa a importância de estudos e desenvolvimento de práticas de GRH com visão mais abrangente da área, buscando lidar também com estímulos que ultrapassam os limites organizacionais e, em última instância, posicionem as características da área em um cenário global. A mudança de contexto, portanto, implica a compreensão mais aprofundada do que a área é, no País, especialmente em sua perspectiva estratégica, que deve estar intimamente relacionada com os resultados organizacionais (Biron, Farndale, \& Paauwe, 2011; Boselie, Dietz, \& Boon, 2005; Delery \& Doty, 1996; Paauwe \& Boselie, 2005).

A importância de conhecer o ambiente institucional em que a GRH se desenvolve ocasionou diferentes estudos internacionais para mapear essas características regionais e proporcionar uma comparação internacional. Esses estudos comumente classificam a área em dois modelos: calculativo e colaborativo (Croucher et al., 2006; Gooderham, Nordhaug, \& Ringdal, 1999; Poutsma, Ligthart, \& Veersma, 2006; Poutsma, Ligthart, Dietz, 2013; Uysal, 2014). Sendo o primeiro predominante nos Estados Unidos e países Anglo-saxões e o segundo em países europeus (Brewster, 2007b). No entanto, ainda é incipiente o número de estudos que problematizam a GRH nacional, inviabilizando a discussão dos sistemas, estratégias e práticas brasileiras em um contexto internacional, solidificando o conhecimento acerca da GIRH (Lazarova, Morley, \& Tyson, 2008).

Nesse sentido, o objetivo deste estudo é analisar a adoção de práticas calculativas e colaborativas, dominantes na gestão internacional comparativa de recursos humanos, segundo os diferentes perfis das áreas de GRH de organizações privadas atuantes no Brasil. Assim, embora este estudo não promova uma comparação de práticas de organizações presentes no Brasil com as de outros países, ele se vale da literatura que subsidia esses estudos comparativos e promove diretrizes iniciais para pesquisas com esse intuito. Além disso, essa pesquisa tem como meta desvenciliar-se do foco sobre os estudos de caso que a perspectiva acadêmica no país possui (Demo, Fogaça, Nunes, Edrei, \& Francischeto, 2011; Tonelli, Caldas, Lacombe, \& Tinoco, 2003).

Esta proposta justifica-se pelos potenciais resultados que a pesquisa pode trazer aos profissionais da área, uma vez que se pretende produzir um cenário mais claro e empiricamente comprovado da GRH, no país. Com isso, será possível tomar decisões mais assertivas sobre a direção que deve orientar suas ações nas organizações. Subramony (2006) chama a atenção desse público, colocando como função crítica da área a demonstração de valores econômicos e credibilidade estratégica, a fim de legitimar a tomada de decisão com base em informações estratégicas e não a partir de percepções subjetivas.

\section{Gestão estratégica de recursos humanos}

Diferentes definições do conceito de GERH (em inglês, Strategic Human Resources Management SHRM) têm sido propostas. Para Martín-Alcázar, Romero-Fernández e Sánchez-Gardey (2005, p. 651), o conceito é visto como "o conjunto integrado de práticas, políticas e estratégias por meio das quais as organizações gerenciam o seu capital humano, que influencia e é influenciado pela estratégia de negócios, pelo contexto organizacional e pelo contexto socioeconômico".

Schuler e Jackson (2005), além de debater o alinhamento vertical, também reconhecem a necessidade de integração entre as práticas da área, conhecida como alinhamento horizontal. Para tanto, os autores acreditam que é adequado mostrar a efetividade da área sobre o desempenho organizacional e a parceria entre os profissionais de $\mathrm{GRH}$ e os gerentes de linha. Nessa perspectiva, a integração entre as políticas da área também fornece as condições que os empregados precisam para alcançar as expectativas almejadas (Demo et al., 2011). A perspectiva de "parceiro" estratégico ganha força, pois o alinhamento horizontal requer um trabalho conjunto das outras gerências e também dos próprios funcionários, bem como práticas efetivas de comunicação.

Kramar e Parry (2014) são mais específicos, ao limitar as características da GRH que efetivamente a tornam estratégica. Primeiro, os autores citam o papel do profissional de GRH na organização, que deve se envolver em decisões estratégicas, além de 
atuar em conjunto com os gerentes de linha. Também destacam que as práticas de capacitação e gestão de desempenho devem estar direcionadas ao alcance dos resultados organizacionais. E, por fim, citam a necessidade de ser estabelecido forte relacionamento com os funcionários e seus representantes, incluindo as entidades sindicais.

A GERH é entendida, neste trabalho, como o conjunto de políticas e práticas propostas para o gerenciamento dos empregados. São desenvolvidas e aplicadas em conjunto com os gerentes de linha; estão integradas entre si, com a estratégia organizacional e ligadas ao resultado da empresa. Para mensurar essa variável, Kramar e Parry (2014, p. 404) propõem:

"A GERH é formada por três áreas: primeiro, o papel do profissional de gestão de recursos humanos, que inclui sua participação na estratégia de negócios, a parceria com os gerentes de linha e avaliação da função de $\mathrm{RH}$; segundo, a gestão de desempenho $e$ capacidade, por meio de sistemas de avaliação, remuneração e formação baseadas no desempenho; e em terceiro lugar, o desenvolvimento de relações diretas com os funcionários por meio de sistemas de comunicação e colaboração".

Essa proposta é compatível com outros trabalhos da área de GERH (Becker, Huselid, \& Ulrich, 2001; Boxall \& Purcell, 2011; Guest, 1989; Lepak \& Shaw, 2008; Schuler, 1992). Assim, é plenamente aceitável que as duas primeiras definições associem o trabalho a ser desenvolvido na área de GRH ao contexto externo, pois é apenas com atenção a esse ponto que a organização terá condições de responder contingencialmente às mudanças ambientais que se apresentarem.

No entanto, se a organização apenas estiver observando o ambiente externo e não conseguir se mobilizar em relação às suas mudanças, o alinhamento vertical será pouco efetivo. Por isso, também precisa promover coesão entre as ações e metas da própria área, que deve funcionar como um sistema integrado no alcance das metas traçadas. A tática auxiliará a organização a adotar as respostas contingenciais mais adequadas e, para tanto, é vital que a área tenha representatividade no mais alto escalão organizacional; mantenha um trabalho em parceria com os gerentes de linha; bem como contribua para o dimensionamento da capacidade interna.

\section{Sistema de gestão internacional comparativa de rh: os modelos calculativo e colaborativo}

A análise da GRH levou alguns autores a criarem tipologias e categorizações daquilo que é praticado pelas organizações á luz de realidades distintas em diferentes países, regiões, setores de atividade e demais fatores institucionais. Assim, dentro da proposta deste estudo, serão abordados os modelos Calculativo e Colaborativo.

A dicotomia entre essas propostas ganha projeção no cenário acadêmico com pesquisadores europeus (Croucher et al., 2006; Gooderham et al., 1999; Poutsma, Ligthart, \& Veersma, 2006; Poutsma et al., 2013; Uysal, 2014). Aos estudiosos questionam a efetividade de práticas de gestão de pessoas da maneira como haviam sido desenvolvidas nos Estados Unidos, pois o contexto europeu era considerado completamente diferente.

O modelo Colaborativo tem cunho mais humanista e vale-se de práticas baseadas em contratos psicológicos estabelecidos entre empregados e empresa, procurando, assim, desenvolver atitudes e comportamentos organizacionais favoráveis (Uysal, 2014). Portanto, os funcionários são vistos como participantes ativos na empresa e a comunicação e cooperação são enfatizadas, caracterizando uma cultura de parceria (Poutsma et al., 2006). Para Gooderham et al. (1999), a premissa almejada com esses empregados é a de compromisso, comunicação e colaboração.

As práticas desse sistema ocorrem por meio da introdução e manutenção de sofisticados dispositivos de relações humanas. Segundo Gooderham et al. (1999), as práticas colaborativas são altamente dependentes do aprimoramento de uma perspectiva menos operacional, envolvendo gestores e especialistas em GRH.

Com essa filosofia, as práticas desse modelo envolvem a comunicação regular, inclusive sobre informações estratégicas, financeiras e organizacionais (Croucher et al., 2006; Gooderham et al., 1999); uso de sistemas de incentivos coletivos (Poutsma et al., 2006); e documentação da missão organizacional e das políticas de emprego (Poutsma et al., 2013). A consulta aos empregados também é bastante recorrente (Gooderham \& Nordhaug, 2010).

Os sindicatos e outras entidades representativas geralmente não criam resistência às práticas 
colaborativas, mas podem colocar pressão sobre a gestão, para que os debates sobre as estratégias ocorram por meio de canais como os conselhos e/ou órgãos de negociação coletiva. Por outro lado, pode haver uma abertura maior entre as questões relativas à negociação e aquelas sujeitas a prerrogativa gerencial, restringindo a participação do sindicato a um aspecto operacional. Também pode haver disposições legais sobre consulta de informações, favorecendo a discussão de assuntos estratégicos pelos órgãos de representação interna (Poutsma et al., 2006).

A GRH Calculativa tem posicionamento racional e baseia-se na hipótese subjacente de que o uso dos recursos individuais, que visam a aumentar os níveis de desempenho, beneficiará a organização como um todo (Poutsma et al., 2006). Por isso, é fundamental uma ligação intrínseca entre empregados e estratégias organizacionais.

O foco reside sobre o exercício consciente dos recursos humanos, pois cada prática utilizada visa à contribuição eficiente dos empregados (Gooderham et al., 1999). Croucher et al. (2006) destacam que as práticas desse sistema são destinadas a alcançar a eficiência em nível individual e envolvem: avaliações e sistemas de recompensas individuais (Croucher et al., 2006; Gooderham \& Nordhaug, 2010; Poutsma et al., 2006); participação financeira dos empregados, por exemplo, inclusive nos lucros (Croucher, Brookes, Wood, \& Brewster, 2010); práticas individuais de desenvolvimento (Gooderham et al., 1999); e avaliação formal dos treinamentos realizados (Poutsma et al., 2013).

Essas práticas são possíveis porque a organização, nesse tipo de modelo, trata cada funcionário como indivíduo e não como membro de uma entidade coletiva protegido por contratos coletivos de emprego e sindicalização. São implantadas por decisão do empregador ou negociadas entre a administração e os trabalhadores, particularmente (Poutsma et al., 2006).

Todavia, embora exista pressão para o emprego de práticas de GRH desenvolvidas e utilizadas nos EUA (Brewster, 2007a, 2007b), estudos em Gestão Comparativa de Recursos Humanos apontam razões para que isso não ocorra. Mesmo que os EUA ainda sejam um grande influenciador da área, as empresas procuram adequar suas práticas às características locais. Esse é o caso da Europa, continente no qual pesquisas apontam a área de GRH com características bem distintas da americana.

Essa diferenciação pode ser expressa por meio da perspectiva estratégica de GRH predominante nessas sociedades. Autores consideram que a perspectiva universalista destaca-se entre os norte-americanos e um posicionamento contextual pode ser identificado entre os europeus (Apospori, Nikandrou, Brewster, \& Papalexandris, 2008; Brewster, 2007a, 2007b). Essa ideia é coerente com a proposição de Müller-Camen (1999), que vê a literatura americana como mais individualista.

Em geral, a literatura em que se apoiam os estudos que diferenciam as características institucionais de GRH na Europa e nos EUA baseiamse em teorias sobre tipos de capitalismo. Os Estados Unidos, bem como o Canadá, Reino Unido e a Austrália, são caracterizados por uma economia de mercado liberal, ou seja, coordenadas primariamente pela competitividade de mercado (Bruzzo \& Basso, 2012; Parry, Dickmann, \& Morley, 2008). Nesses países, há um foco no individualismo e os sindicatos têm pouca influência nas relações de trabalho. Por isso, práticas relacionadas à gestão de desempenho e recompensas encontram maior abertura (Brookes, Croucher, Fenton-O'Creevy, \& Gooderham, 2011).

Já nos países europeus de origem não anglo-saxã (Alemanha, Suécia, Dinamarca e Holanda) predominam economias coordenadas de mercado. Portanto, mantêm uma interação mais estratégica com o governo, os bancos, as universidades, entidades da indústria, os sindicatos e as federações de empregadores. Nesses países, há interferência nas relações de trabalho, nos modos de atividades de investimento e governança corporativa (Hall \& Soskice, 2001; Uysal, 2014; Whitley, 1999).

Assim, o modelo americano de GRH desenvolveuse em um cenário menos regulamentado, no qual as organizações se preocupavam com resultados mensuráveis e, para isso, procuravam determinar as práticas mais efetivas na obtenção desses resultados. Já o contexto europeu é completamente diferente. As variáveis contextuais teriam peso muito maior na regulamentação dos negócios (Brewster, 2007b). Haja vista que a maioria dos países da Europa é membro da União Europeia (UE), que apresenta como tendência a integração política, econômica e social, e tem como objetivo liberalizar o fluxo de bens, serviços, pessoas e capitais (Mayrhofer, Brewster, Morley, \& Ledolter, 2011). 
Brewster (2007b) faz um estudo mais aprofundado com o objetivo de comparar as características específicas da área e dos estudos sobre GRH entre essas regiões. Os resultados sintetizados desse estudo estão sumarizados na Tabela 1.

Tab. 1

Comparação das características

da GRH entre Estados Unidos e Europa.

\begin{tabular}{ccc}
\hline $\begin{array}{c}\text { Características } \\
\text { de Análise }\end{array}$ & Estados Unidos & Europa \\
\hline Foco do RH & $\begin{array}{c}\text { Desempenho } \\
\text { na empresa }\end{array}$ & $\begin{array}{c}\text { Empresa dentro } \\
\text { de um contexto }\end{array}$ \\
\hline Tipo de literatura & Prescritiva & Crítica \\
\hline $\begin{array}{c}\text { Perspectiva } \\
\text { metodológica }\end{array}$ & Dedutiva & Indutiva \\
\hline $\begin{array}{c}\text { Características } \\
\text { culturais }\end{array}$ & Individualista & Mais coletivista \\
\hline $\begin{array}{c}\text { Legislação de } \\
\text { emprego }\end{array}$ & $\begin{array}{c}\text { Autorregulação } \\
\text { do mercado }\end{array}$ & $\begin{array}{c}\text { Forte controle } \\
\text { do Estado }\end{array}$ \\
\hline $\begin{array}{c}\text { Sindicatos } \\
\text { Menos }\end{array}$ & $\begin{array}{c}\text { Presença } \\
\text { maciça }\end{array}$ \\
\hline
\end{tabular}

Fonte: Construído com base no estudo de Brewster (2007b).

Assim, verifica-se que a GRH americana é mais calculativa, pois enfatiza o mercado externo, os papeis individuais e a prestação de contas, do que as relações estabelecidas nesse contexto (Brookes et al., 2011). Já na Europa, a configuração mais comumente encontrada é a colaborativa, na qual as divergências de interesses são reconhecidas, mas existe a obrigação de integrar os grupos por uma série de mecanismos, incluindo a comunicação intensiva (Gooderham et al., 1999). Isso significa que, no primeiro caso, o interesse reside sobre os shareholders, enfatizando o mercado, enquanto no segundo os interesses recaem sobre os stakeholders, que precisam ser atendidos, enfatizando as relações deles com as empresas.

Por fim, cabe salientar que, embora as abordagens Calculativa e Colaborativa constituam dois conjuntos distintos, as práticas de GRH não devem ser concebidas como representantes de duas extremidades diferentes de um continuum, mas como ortogonais (Gooderham et al., 1999), visto que esses modelos não são opostos e que em algum momento se interceptam. Rousseau e Arthur (1999) classificam esses posicionamentos como complementares.

Croucher et al. (2006) destacam que a escolha por um ou outro sistema não é exatamente das organizações e suas respectivas áreas de gestão de pessoas, mas consequência da influência do contexto nacional e, em particular, das instituições do sistema nacional de relações industriais. Os determinantes institucionais têm forte efeito sobre a aplicação de práticas de gestão de pessoas em ambos os modelos (Gooderham et al., 1999).

\section{Metodologia}

Utilizou-se uma abordagem quantitativa de cunho descritivo transversal (Babbie, 2001; Malhotra, 2006). O método empregado foi do tipo Survey, operacionalizado por meio de um questionário com questões fechadas versando sobre práticas de GRH e características organizacionais, desenvolvido pelo The Cranfield Network on International Human Resource Management (Cranet). Trata-se de uma rede de pesquisa internacional focada na comparação de práticas em diferentes países e que viabiliza, simultaneamente, a compreensão das características de gestão locais.

O questionário versa sobre práticas de gestão de pessoas e características organizacional e foi submetido à técnica de translation/back-translation (Cranet, 2011). A escolha desse instrumento deveuse ao seu reconhecimento acadêmico internacional, pois vem sendo utilizado, há anos, em diferentes pesquisas na área de gerenciamento de pessoas (Bruzzo \& Basso, 2012; Gooderham \& Nordhaug, 2010; Gurkov, Zelenova, \& Saidov, 2012; Larsen \& Brewster, 2003; Rizov \& Croucher, 2009; Stavrou \& Kilaniotis, 2010; Supangco, 2012). Além disso, como a proposta deste estudo também é posicionar as características estratégicas da GRH identificadas em uma perspectiva internacional, o uso de um instrumento validado e utilizado em outros países viabilizaria comparações mais assertivas.

A população-alvo deste estudo foi composta de empresas privadas que empregavam cinco ou mais funcionários no ano de 2014, período da realização da pesquisa empírica, e acessada por um procedimento de amostragem não probabilístico. $\mathrm{O}$ responsável pelo preenchimento do questionário deveria estar envolvido no gerenciamento de pessoas na empresa, pois precisavam ter um conhecimento amplo da área.

Inicialmente, cinco deles participaram de um préteste para verificar a usabilidade do site no qual o questionário estava hospedado e a compreensão da tradução proposta. Posteriormente aos ajustes indicados no pré-teste, foram enviados 22.052 e- 
mails aos responsáveis pela área de gerenciamento de pessoas de empresas com atuação no Brasil e 1.295 (5,9\%) organizações atenderam ao pedido de participação na pesquisa. No entanto, o montante de 862 participações (66,5\% do total de respondentes) foram descartados por não terem completado o preenchimento do questionário, ou por apresentarem quantidade muito elevada de respostas incompletas.

Restaram 433 participações válidas. A depuração da base de dados levou à exclusão de todos os respondentes que não atuam no setor privado, tinham menos de cinco funcionários e instituições que não possuíam uma área de GRH formalizada e, portanto, não atendiam aos objetivos deste estudo. Por fim, a amostra total utilizada para atender aos objetivos propostos somou 326 organizações.

As respostas dos participantes foram armazenadas em um banco de dados e transferidas para o SPSS 19.0 (Statistical Package for Social Sciences), software utilizado para promover a análise estatística dos achados do estudo. Inicialmente foram realizadas análises estatísticas descritivas (média, desvio padrão e frequência) para caracterizar a amostra. Posteriormente, a Gestão de Recursos Humanos das organizações pesquisadas foi classificada em diferentes grupos por meio de uma análise de agrupamentos. Por fim, foi realizada uma Análise de Variância (Anova) para estudar as diferenças entre médias dos grupos formados na etapa anterior quanto a práticas calculativas e colaborativas.

\section{Análise dos resultados}

\subsection{Caracterização da amostra}

O número de funcionários encontrados entre os respondentes variou de 7 até 100.489. A média foi de 3.387,40 funcionários, mas, como consequência dessa amplitude tão elevada, o desvio padrão foi alto (DP = 10.999,19).

Com relação ao setor de atuação das empresas pesquisadas, a análise desagregada dos dados apresenta amostra bastante heterogênea, sugerindo contextos, tecnologias e públicos distintos. Entre os destaques, encontra-se o predomínio dos comércios varejista e atacadista (10,1\%); seguidos pelas áreas de manufatura de comida; bebidas; têxteis; madeira e papel; coque; petróleo refinado; e produtos relacionados $(8,9 \%)$. É necessário destacar também a representatividade da área de Tecnologia da Informação (TI), com 7,7\%. Por outro lado, alguns tipos de serviços (acomodação e alimentação; publicação; atividade de radiodifusão), com 0,9\%; e manufatura (de computador, produtos eletrônicos e equipamentos elétricos e de equipamentos de transporte), com 2,4\%, foram os menos representados na pesquisa.

Outra importante característica investigada foi o continente de localização da sede organizacional, apresentado na Tabela 2.

Tab. 2

Distribuição das empresas pesquisadas

por continente em que a sede está localizada.

\begin{tabular}{|c|c|c|}
\hline Continente & $n$ & $\%$ \\
\hline Ásia* & 9 & 3 \\
\hline América do Norte & 24 & 7 \\
\hline Europa & 40 & 12 \\
\hline América do Sul & 253 & 78 \\
\hline
\end{tabular}

Fonte: Elaboração própria a partir da coleta de dados da pesquisa Nota: $\mathrm{n}=$ número absoluto de respondentes; $\%$ = porcentagem. *Turquia foi incluída no continente asiático.

Com exceção da Oceania, todos os outros continentes estão representados na amostra. O número menor de sedes pertence à Ásia, seguido pela América do Norte, e a maioria das 24 referências aponta a sede em território norte-americano. $\mathrm{Na}$ Europa, que contempla $12 \%$ das sedes de empresas pesquisadas, não houve predomínio de nenhum país de forma distinta. Por fim, embora algumas sedes estejam na América do Sul, a maioria das empresas $(247 ; 75,8 \%)$ caracterizada nesse segmento tem sede no Brasil. Nesse sentido, tem mais coerência que o predomínio do mercado de atuação dessas empresas seja nacional.

Embora não sejam representativos da população, os dados de caracterização da amostra revelam aspectos bastante heterogêneos e que, de certa forma, correspondem às características do cenário nacional. No entanto, além de caracterizar os respondentes desta pesquisa para posterior ampliação dos resultados alcançados, tais aspectos que podem influenciar a gestão de pessoas das organizações.

Quanto à região demográfica brasileira na qual a empresa se encontra, sabe-se que a cultura, as variáveis institucionais e a capacidade de produção de riqueza dos estados são diferentes. Consequentemente, é de se esperar que os esforços 
no direcionamento do comportamento das pessoas no trabalho se adéquem também para atender à especificidades regionais. A sede organizacional traz implicações sobre a transparência das práticas e a busca de resultados organizacionais, bem como um posicionamento mais estratégico, a fim de se manter mais competitivas.

Já o tamanho da organização pode afetar diferentes aspectos, como o nível de formalização e comunicação de práticas; a profissionalização das ações e dos profissionais da área de $\mathrm{GRH}$; e, até mesmo, a distribuição de tarefas na área. O setor de atuação também é de fundamental importância para a compreensão dos aspectos estratégicos estudados. Atuar em um cenário mais competitivo, como na área de $\mathrm{TI}$, pode implicar o uso de práticas mais focadas em resultados e valorização das pessoas para reter profissionais. Já para setores de commodities, as necessidades podem ser completamente diferentes.

\subsection{Classificação dos diferentes perfis das áreas de} $\mathrm{GRH}$ de organizações privadas atuantes no Brasil

Considerando que o foco de análise deste estudo reside sobre os diferentes perfis, decidiu-se realizar o agrupamento das organizações por perfil estratégico adotado. Para esse agrupamento foi realizada uma análise de cluster a partir três critérios defendidos por Kramar e Parry (2014) e apresentados previamente na literatura:

1. Papel da GRH na estratégia organizacional;

2. Gestão do desempenho e da capacidade dos empregados da organização;

3. Relacionamento com empregados e seus representantes.

Julgou-se importante testar a consistência interna dos indicadores propostos para a formação de cada variável. Com o uso do teste Alfa de Cronbach, decidiu-se eliminar quatro conjuntos de itens: 1. Atuação conjunta entre GRH e gerentes de linha na tomada de decisões; 2. Missão e estratégia organizacional escritas; 3. Proporção de empregados sindicalizados; e 4. Extensão da influência dos sindicatos na organização. A exclusão dessas variáveis gerou um resultado satisfatório no teste de confiabilidade $(\alpha<0,6)$.

Selecionadas as variáveis para a análise de aglomerados, realizou-se a checagem de outliers e multicolinearidade. Segundo Fávero, Belfiore, Silva, \& Chan (2009), cluster analysis é uma técnica sensível a esses aspectos que, quando existem, tendem a distorcer os resultados. Quanto aos outliers, nenhuma resposta ou respondente enquadrou-se nesse quesito. A multicolinearidade foi mensurada por meio da análise de correlação de Pearson e, embora todas tenham sido significativas $(p>0,001)$, nenhuma configurou-se como altamente correlacionadas entre si (coeficiente $p>0,7$ ), ou seja, sem sobreposição de variáveis.

Atendidos os pré-requisitos, a análise de cluster propriamente dita foi realizada por meio de dois métodos de agrupamentos distintos, mas complementares. No primeiro momento, utilizou-se a Análise de Conglomerados Hierárquicos com método de análise Ward, que coloca cada caso como um agrupamento separado e, posteriormente, vai fazendo combinações sequenciais hierárquicas de grupos aninhados até incluir todos em um único grupo (Antonenko, Toy, \& Niederhauser, 2012). Nessa análise utilizou-se o método $Z$ score para a padronização das medidas e o dendograma para extrair o melhor número de agrupamentos.

A melhor configuração resultou na formação de quatro grupos distintos. Isso serviu de base para a segunda análise, na qual se utilizou um algoritmo não hierárquico ( $K$ means) para a classificação dos respondentes em quatro grupos. A Anova resultante dessa análise validou a construção dos grupos propostos e é mostrada na Tabela 3.

Tab. 3

Análise da variância (Anova)

\begin{tabular}{|c|c|c|c|c|c|c|}
\hline \multirow[b]{2}{*}{ Variáveis } & \multicolumn{2}{|c|}{ Cluster } & \multicolumn{2}{|c|}{ Erro } & \multirow[b]{2}{*}{$\mathrm{F}$} & \multirow[b]{2}{*}{ Sig. } \\
\hline & $\begin{array}{l}\text { Quadrado } \\
\text { Médio }\end{array}$ & $\mathrm{GL}$ & $\begin{array}{l}\text { Quadrado } \\
\text { Médio }\end{array}$ & $\mathrm{GL}$ & & \\
\hline 1. Papel do RH na estratégia organizacional & 76,506 & 3 & ,297 & 322 & 258,002 & ,000 \\
\hline 2. Gestão do desempenho e da capacidade da mão de obra & 57,946 & 3 & ,469 & 322 & 123,436 & ,000 \\
\hline 3. Relacionamento com empregados e representantes & 76,800 & 3 & ,294 & 322 & 261,414 & ,000 \\
\hline
\end{tabular}

Fonte: Elaboração própria a partir da coleta de dados da pesquisa. Nota: GL = Graus de liberdade 
Pelos dados resultantes, verifica-se que as variáveis consideradas influenciaram na separação dos grupos, pois todas apresentaram $p<0,001$. Outro dado importante observado é o valor estatístico de $F$, que classifica as variáveis no processo de separação. Pelos números resultantes, a variável que mais contribuiu para a diferenciação dos grupos foi o relacionamento com os empregados, seguida pelo papel da GRH na estratégia organizacional e, por último, a gestão do desempenho e da capacidade dos empregados. Com isso, buscou-se delimitar o perfil de cada cluster formado avaliando-se a distância entre os centroides dos clusters formados. Esse resultado é relatado na Tabela 4. avaliação formal de desempenho e pouca associação entre resultados e recompensas; relativamente boa comunicação estratégica.

Claramente, ainda existem, no Brasil, empresas que possuem um departamento focado na gestão de pessoas, mas não mantem ligação com a estratégia organizacional; não avalia ou recompensa contingencialmente os empregados; e também não viabiliza uma comunicação assertiva, sugerindo a existência de uma GRH meramente burocrática e processual, que realmente tem pouco a contribuir para resultados superiores, pois estão, no máximo, preocupadas em seguir a "cartilha" da área.

Tab. 4

Distância final entre os centroides dos clusters formados

\begin{tabular}{|c|c|c|c|c|}
\hline \multirow{2}{*}{ Variáveis } & \multicolumn{4}{|c|}{ Clusters } \\
\hline & 1 & 2 & 3 & 4 \\
\hline Zscore: Papel do RH na estratégia organizacional & $-1,27539$ & 0,83597 & 0,47816 & $-0,6565$ \\
\hline Zscore: Gestão do desempenho e da capacidade da mão de obra & $-0,83065$ & 0,92607 & 0,12846 & $-0,76508$ \\
\hline Zscore: Relacionamento com empregados e representantes & $-1,28638$ & 1,01843 & $-0,3456$ & 0,20846 \\
\hline $\mathrm{n}$ & 67 & 102 & 91 & 66 \\
\hline$\%$ & 20,55 & 31,29 & 27,91 & 20,25 \\
\hline
\end{tabular}

Fonte: Elaboração própria a partir da coleta de dados da pesquisa.

Com base nesses dados, foi proposta a existência de quatro grupos estratégicos de GRH nas organizações pesquisadas:

1. GRH Operacional: baixa formalização das práticas de GRH e integração organizacional; pouco uso de avaliação formal de desempenho e pouca associação entre resultados e recompensas; comunicação estratégica deficitária.

2. GRH Estratégica: alta formalização das práticas de GRH e integração organizacional; uso frequente da avaliação formal de desempenho e associação entre resultados e recompensas; boa comunicação estratégica.

3. GRH Formalizada: relativamente alta formalização das práticas de GRH e integração organizacional; ligeiramente alto uso da avaliação formal de desempenho e associação entre resultados e recompensas; comunicação estratégica deficitária.

4. GRH Comunicativa: relativamente baixa formalização das práticas de GRH e integração organizacional; pouco uso de
Por outro lado, destaca-se o grande número de empresas classificadas no grupo estratégico, conjunto com maior número de organizações, o que pode levar a duas possíveis constatações. A primeira é que a amostragem por conveniência favoreceu a participação de empresas mais estruturadas, portanto, com práticas mais avançadas de GERH. A segunda é que realmente existe considerável número de empresas no País que adota práticas estratégicas de GRH. Confirma-se com essa classificação a suposição de diferentes perfis estratégicos de gestão de pessoas nas empresas pesquisadas. Portanto, mesmo não consideradas plenamente estratégicas, muitas empresas podem estar se direcionando nesse sentido.

5.3 Comparação de práticas calcutativas e colaborativas entre os grupos de GERH das empresas pesquisadas

Nesta última análise proposta, o intuito foi investigar a adoção de práticas calculativas e colaborativas entre os quatro grupos estratégicos delineados na análise anterior (Croucher et al., 2010, 2006; Gooderham et al., 1999, 2008; Poutsma et al., 2006). 
O primeiro ponto analisado foi a confiabilidade do uso conjunto dos itens escolhidos, mensurados por meio do Alfa de Cronbach. A única variável que teve itens excluídos para adequação da confiabilidade foi Mensuração da Efetividade do Treinamento, que ao final foi mensurada por: Cumprimento dos Objetivos Definidos no Plano de Treinamento e Desenvolvimento; Mensuração do Desempenho no Trabalho Antes e Imediatamente depois do Treinamento; Mensuração do Desempenho no Trabalho antes e alguns Meses depois do Treinamento; Feedback Informal dos Gerentes de Linha e Feedback Informal dos Empregados.

Com a definição dessas variáveis, realizou-se a comparação das médias por meio de uma Anova. Os resultados dessa análise são mostrados na Tabela 5.

Para a análise desses dados, foi importante considerar que, primariamente, a Análise de Variância indica apenas se existe ou não diferença entre os grupos considerados. Para identificar onde essas diferenças se encontram foi necessário realizar um teste post-hoc, sendo escolhido para este estudo o Games-Howell e Bonferroni. O primeiro para variâncias iguais e, o segundo, para diferentes.

O primeiro dado observado na Tabela 5 é a variável RH Trabalha com os Gestores de Linha que não apresenta diferença significativa entre os grupos pesquisados. Isto sugere que essa é uma prática difundida entre as organizações, independentemente de como a gestão de pessoas dessas empresas trabalha. Uma explicação plausível para isso é que o personalismo da cultura brasileira pode atuar de forma mais contundente nesse ponto das organizações do que a estratégia adotada. Todas as outras variáveis apresentaram diferença em pelo menos uma das comparações de grupo. Na análise das variáveis categorizadas como calculativas (quatro primeiras linhas da Tabela 4), o grupo GRH Operacional apresenta diferença significativa em todas as variáveis dessa categoria, quando comparado ao grupo GRH Estratégico e, para as três primeiras variáveis do grupo GRH Formalizado. Assim, analisando as médias, é possível dizer que o GRH Operacional é menos calculativo que esses dois grupos, pois todas as suas médias são inferiores. Além disso, não foi encontrada diferença significativa entre os grupos GRH Operacional e Comunicativo para as variáveis calculativas.

Comparando o grupo GRH Estratégico com a GRH Formalizada, encontrou-se diferença estatística

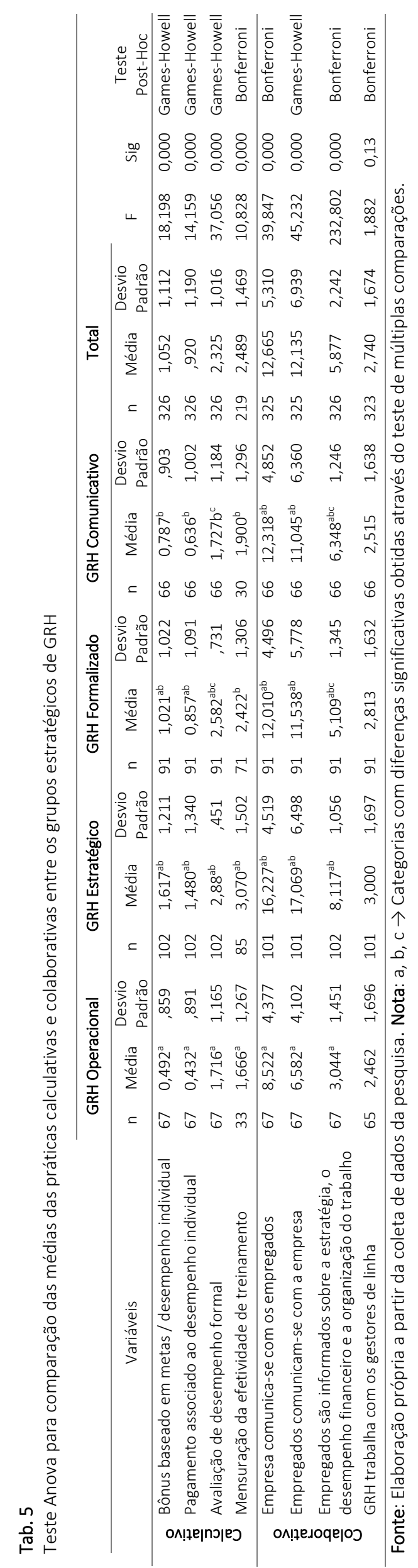

Internext | São Paulo, v.11, n. 2, p. 49-65, mai./ago. 2016 
significativa, em todas as variáveis. O mesmo ocorreu no grupo GRH Comunicativa, exceto para a variável Avaliação de Desempenho Formal. Dessa forma, novamente constata-se que, em todos esses casos, a média obtida pelo grupo GRH Estratégico para as variáveis calculativas foi maior do que nos demais grupos analisados.

A única diferença estatística significativa entre a GRH Formalizada e a Comunicativa aconteceu para a variável Avaliação de Desempenho Formal. A média do primeiro grupo foi significativamente maior do que a do segundo. Isso sugere que o grupo GRH Formalizada é mais calculativo do que o grupo GRH Comunicativa.

Desse modo, o que se delineia, é que a tipologia calculativa sobressai nas organizações que apresentam gestão de pessoas mais estratégica. $O$ segundo grupo mais Calculativo foi a GRH Formalizada e não se evidenciou diferença significativa no uso dessas práticas entre os grupos GRH Operacional e Comunicativa.

Com relação à perspectiva colaborativa, foram consideradas apenas as três primeiras variáveis, para essa análise comparativa: Empresa Comunica-se com os Empregados; Empregados Comunicam-se com a Empresa; e Empregados São Informados sobre a Estratégia, Desempenho Financeiro e Organização do Trabalho. O grupo GRH Operacional apresentou diferença significativa quando comparado a todos os demais e, novamente, as menores médias. Dessa forma, pode ser considerado o menos colaborativo entre os grupos.

A GRH Estratégica também apresentou as maiores médias para as três variáveis consideradas quando comparada a todos os demais grupos. Assim, além de ser o grupo mais calculativo, é composto por empresas que também mais empregam práticas colaborativas.

O grupo GRH Formalizada apresentou diferença quando comparado ao da GRH Comunicativa apenas para o item Empregados são informados sobre a Estratégia, Desempenho Financeiro e Organização do Trabalho, com a média da primeira menor do que a da segunda. Desse modo, o grupo GRH Comunicativa apresenta mais características colaborativas do que o da GRH Formalizada, perdendo apenas para a Estratégica.

Com relação aos achados das práticas colaborativas, destacou-se novamente o grupo estratégico. Porém, o segundo lugar foi ocupado pelo grupo GRH Comunicativa, seguido por GRH Formalizada e Operacional, respectivamente.

Desse modo, verificou-se que a perspectiva estratégica organizacional tem relação com as práticas de gestão de pessoas delimitadas. Depois, também é importante destacar que, corroborando a divisão resultante da análise de agrupamentos e do perfil de classificação dessas empresas, os grupos GRH Formalizada e GRH Comunicativa, aproximam-se da GRH Estratégica, e cada um favorece um grupo de práticas e posicionamento diferentes, o que poderia ser visto como uma fase de transição das áreas de $\mathrm{GRH}$ nesses grupos.

O mais expressivo desses resultados, no entanto, foi o fato de o grupo estratégico ter sido classificado pela adoção tanto de práticas mais calculativas quanto colaborativas. Embora seja possível (Gooderham et al., 1999), o que se verifica é o destaque de um ou outro conjunto de práticas por região investigada. Uma explicação conjunta para esse fato é que as práticas calculativas atuam no nível individual e as colaborativas grupalmente. Além disso, a pluralidade associada ao cenário brasileiro, construído a partir de diferentes fontes, pode explicar a prevalência desses dois posicionamentos na perspectiva estratégica.

Da disposição calculativa, pode-se destacar o predomínio da influência da literatura americana na área de GRH no País. Além disso, a existência, no País, de multinacionais com sede em países nos quais essas práticas predominam, também pode favorecer a sua adoção e legitimação. Culturalmente, a plasticidade, ou seja, valorização do que é estrangeiro, também traz mais propensão em assumir modelos e conceitos desenvolvidos em países com predomínio dessas práticas, especialmente os EUA.

Por outro lado, várias características da cultura brasileira favorecem as práticas colaborativas: coletivismo, personalismo, feminilidade e cordialidade. Esses aspectos sustentam ações que consideram a visão dos empregados, a comunicação e a parceria com os empregados (Croucher et al., 2006). No mais, as relações de trabalho são reguladas por uma legislação que garante variados direitos aos trabalhadores, bem como sua proteção. Os sistemas legais adotados no Brasil restringem a possibilidade de práticas calculativas de GRH (Brookes et al., 2011). 
A interpretação desses achados requer lembrar que a escolha de um ou outro conjunto de práticas não é decisão autônoma da GRH das empresas pesquisadas, mas está alinhada com a organização e são influenciadas, principalmente, pelo contexto local, com as particularidades institucionais existentes (Croucher et al., 2006). Nesse sentido, pesquisas que façam essa contextualização poderão trazer resultados promissores sobre os fatores motivadores da adoção de diferentes modelos de gestão de pessoas no Brasil.

\section{Considerações finais}

A GRH pode criar condições organizacionais que alavancam a competitividade, mas isso depende de suas escolhas e de adequado posicionamento. Requer, assim, conhecimento sobre a área e compreensão do contexto no qual ela se insere (Martín-Alcázar et al., 2005). O campo contextual engloba, além da própria organização, os cenários local e global, que podem exercer potencial influência sobre a escolha das práticas de gerenciamento de pessoas pelos gestores.

Todos esses cenários têm sofrido mudanças, nas últimas décadas, e pressionado a GRH a modificar o modo como as empresas gerenciam seus empregados. A literatura que investiga essa área, no Brasil, relata um movimento que transita do posicionamento operacional para uma tendência estratégica (Coltro, 2009; Fischer, 2002; Lacombe \& Tonelli, 2001; Piellusch \& Taschner, 2009; S. Silva \& Azzuz, 2003; Tinoco, 2005).

Tal constatação reforça a importância de compreender a dispersão das práticas adotadas pela GRH nas empresas que atuam no Brasil. De modo geral, os dados apresentados neste estudo sobre a GRH no Brasil corroboram a literatura (Tanure, Evans, \& Cançado, 2010b; Tanure, Evans, \& Pucik, 2007; I. Vasconcelos, Mascarenhas, \& Vasconcelos, 2004) que sugere que a área, no País, vive um período de transformação, guiando-se para uma perspectiva estratégica. Há maior concentração de empresas no grupo GRH Estratégica, seguido pelos GRH Formalizada e Comunicativa, que apresentam algumas práticas também caracterizadamente estratégicas.

Essa classificação permitiu relacionar esses grupos aos modelos calculativo e colaborativo (Croucher et al., 2010, 2006; Gooderham et al., 1999, 2008;
Poutsma et al., 2006). As empresas classificadas no grupo GRH Estratégica têm práticas mais calculativas e colaborativas do que as demais empresas. Por outro lado, as empresas classificadas como GRH Operacional apresentam médias menores para todas as práticas e em relação a todos os grupos que possuem diferenças significativas. Os grupos GRH Formalizada e GRH Comunicativa, com médias significativamente diferentes dos outros grupos (Operacional e Estratégico), apresentaram valores intermediários. Entre si, obtiveram diferença apenas em um dos itens de cada conjunto de práticas. Assim, as empresas da GRH Formalizada puderam ser classificadas como mais calculativas e aquelas pertencentes à GRH Comunicativa, como mais colaborativas. Embora nenhuma delas tenha atingido médias superiores à da GRH Estratégica.

A compreensão desses achados requer a discussão sobre os fatores institucionais e culturais que interferem tanto nas escolhas realizadas pelas organizações quanto nas práticas de GRH implantadas (Brewster, 2004; Brewster, 2006; Budhwar \& Debrah, 2001; Budhwar \& Sparrow, 2002; Gooderham \& Nordhaug, 2010; Morley \& Collings, 2004; Müller-Camen, 1999; Tanure et al., 2007). Determinadas condições podem favorecer ações calculativas, enquanto outras razões podem estimular práticas colaborativas.

Como já apresentado, a aceitação e incorporação da literatura americana no Brasil tende a favorecer a escolha de práticas calculativas, pois a cultura dos EUA é tida como individualista e masculina (competitiva) (G. Hofstede, G. J. Hofstede, \& Minkov, 2010; Hofstede, 2001). Outro ponto importante é a convivência de organizações de diferentes origens no mesmo contexto. A amostra estudada era composta por $25 \%$ de organizações originárias de outros países. A presença de organizações de origem americana também pode reforçar a adoção de práticas calculativas. Por outro lado, filiais com sede na Europa tendem a favorecer práticas colaborativas, uma vez que Brewster (2007b) classifica a GRH nessa região como mais coletivista, com controle do Estado sobre as relações de trabalho e uma literatura mais crítica.

Na perspectiva nacional, a cultura brasileira é tida como coletivista (Barbosa, 2003; Hofstede, et al., 2010; Hofstede, 2001), ou seja, há especial foco nos relacionamentos, na harmonia do grupo e controle de confronto, bem como alcance das metas 
estabelecidas para o grupo. Além disso, o País é marcado por grandes desigualdades, e por isso, "desenvolve compensatoriamente um sistema de proteção social amplo" (Bresser-Pereira, 2011, p.10).

Essas variáveis formam um quadro bastante heterogêneo, pois variáveis antagônicas pressionam as organizações situadas no País em diferentes direções. Isto torna compreensível a adoção de variadas práticas de GRH pelos grupos delineados. As empresas classificadas como GRH Estratégica parecem ser as únicas que conseguem trabalhar com esses distintos posicionamentos, valendo-se tanto de ações de cunho calculativo quanto colaborativo. Isso ratifica a literatura que apresenta essas abordagens distintas não como excludentes, mas como ortogonais (Gooderham et al., 1999) ou complementares (Rousseau \& Arthur, 1999).

Esses achados ainda corroboram a pesquisa de Lemos, Santos e Dubeux (2013, p. 81), na qual investigam "as expectativas de trabalhadores brasileiros e norte-americanos acerca de diretrizes de recursos humanos das organizações". Na pesquisa empírica realizada, identificou-se a valorização de práticas focadas no grupo, independentemente da nacionalidade do respondente, e aceitação ligeiramente maior dos brasileiros pelo uso de práticas ligadas a aspectos individuais. Esses dados reforçam a importância das organizações desenvolverem ações capazes de conciliar práticas de linhas distintas, tanto calculativas como colaborativas.

Outras análises decorrentes desta investigação confirmam que as práticas do grupo classificado como Operacional foram as menos calculativas e colaborativas, sugerindo que essas empresas estão aquém das demais para lidar com as forças que as pressionam tanto para a eficiência quanto para o relacionamento com os empregados. Nesse mesmo sentido, pode-se dizer que os grupos GRH Formalizada e Comunicativa se destacaram, respectivamente, nas práticas calculativas e colaborativas, embora em menor grau do que a GRH Estratégica. Portanto, os dados sugerem que as empresas desses grupos estão mais preparadas para lidar com as forças institucionais e culturais que agem sobre a gestão de pessoas do que as empresas classificadas como Operacionais e que parecem ter predisposições distintas para lidar com tais fatores.

Com base nesses achados, é possível afirmar que a evolução da área para uma perspectiva mais estratégica sugere a capacitação das organizações para responder às demandas atuais, pois se sabe que a área de gestão de pessoas tem influência sobre o sucesso organizacional, embora não seja a única condição para isso. Para que seu desenvolvimento continue avançando, é importante que aspectos revelados mais incipientes ganhem novos contornos, como o desenvolvimento e a aplicação de práticas que relacionem desempenho e recompensas e medidas mais efetivas dos resultados proporcionados pelos treinamentos aplicados pelas empresas.

Outro ponto de destaque é o uso conciliatório de práticas de gestão de pessoas voltadas para o desempenho (calculativas) quanto à colaboração. A miscigenação histórica e cultural brasileira permite esse uso conjunto de práticas que têm as mesmas origens. Ao contrário das organizações europeias, em que predominam práticas colaborativas, e as originárias dos países anglo-saxões, em que é preponderante a GRH Calculativa, as empresas no Brasil adotam ambas.

\subsection{Limitações do estudo e sugestões para pesquisas} futuras

Inevitavelmente, qualquer pesquisa, por envolver escolhas, sofre limitações. Neste estudo é importante destacar que, diferente de outras pesquisas da área, esta não trabalhou com a percepção dos empregados da GRH, aspecto que pode ser favorável, pois diminui a parcialidade na obtenção dos resultados. Por outro lado, traz uma visão "fria" sobre o que acontece nas organizações, pois analisa a existência ou não de determinadas práticas, não permitindo uma análise aprofundada de como essas práticas são efetivamente realizadas.

Também é necessário destacar que, embora a amostra tenha sido expressiva, não é possível generalizar os dados relatados para todo o Brasil, pois não é representativa desse cenário. Para minimizar esse problema, sugere-se que pesquisas futuras ampliem o tamanho da amostra e o método de amostragem empregado.

Entre sugestões para pesquisas futuras, recomenda-se a investigação do conjunto de práticas de GRH ditas coletivas (Poutsma et al., 2013; Rizov \& Croucher, 2009), que coloca os funcionários como detentores do conhecimento organizacional para produzir resultados mais efetivos. Outra possibilidade, considerando ainda esse aspecto, é investigar se as práticas calculativas e colaborativas 
expressam-se da mesma forma para todas as camadas organizacionais. Explorar em profundidade essa concepção pode trazer conciliação à ideia da existência conjunta dessas ações de gestão de pessoas, que, embora não sejam incompatíveis, cresceram de raízes distintas.

Recomenda-se a comparação dos dados encontrados nesta pesquisa com os apresentados por outros países.

\section{Referências}

- Antonenko, P. D., Toy, S., \& Niederhauser, D. S. (2012). Using cluster analysis for data mining in educational technology research. Educational Technology Research and Development, 60, 383398. doi: 10.1007/s11423-012-9235-8

- Apospori, E., Nikandrou, I., Brewster, C., \& Papalexandris, N. (2008). HRM and organizational performance in northern and southern Europe. The International Journal of Human Resource Management, 19(7), 1187-1207.

- Babbie, E. (2001). Métodos de Pesquisa de Survey. Belo Horizonte: Editora UFMG.

- Barbosa, L. (2003). Igualdade e meritocracia: a ética do desempenho nas sociedades modernas (4 ed.). Rio de Janeiro: Editora FGV.

- Becker, B. E., Huselid, M. A., \& Ulrich, D. (2001). Gestão estratégica de pessoas com scorecard: interligando pessoas, estratégias e performance. Rio de Janeiro: Campus.

- Biron, M., Farndale, E., \& Paauwe, J. (2011). Performance management effectiveness: lessons from world- leading firms. The International Journal of Human Resource Management, 22(06), 1294-1311. doi: 10.1080/09585192.2011.559100

- Boselie, P., Dietz, G., \& Boon, C. (2005). Commonalities and contradictions in research on Human Resource Management and performance. Human Resource Management Journal, 15(3), 6794. doi: 10.1111/j.1748-8583.2005.tb00154.x

- Boxall, P., \& Purcell, J. (2011). Strategic and Human Resource Management (3rd ed.). London: Palgrave Macmillan.

- Bresser-Pereira, L. C. (2011). Cinco modelos de capitalismo. Texto Para Discussão EESP/FGV, (280), 1-17.

- Brewster, C. (2004). European perspectives on Human Resource Management. Human Resource Management Review, 14, 365 - 382.
- Brewster, C. (2006). Comparing HRM policies and practices across geographical borders. In G. K. Stahl \& I. Björkman (Eds.), Handbook of Research in International Human Resource Management (1st. ed.). Cheltenham: Edward Elgar Publishing.

- Brewster, C. (2007a). A European perspective on HRM, European J. International Management, 1(3), 239-259.

- Brewster, C. (2007b). Comparative HRM: European views and perspectives. The International Journal of Human Resource Management, 18(5), 769-787. doi:10.1016/j.sbspro.2012.09.1024

- Brookes, M., Croucher, R., Fenton-O'Creevy, M., \& Gooderham, P. (2011). Measuring competing explanations of Human Resource Management practices through the Cranet survey: cultural versus institutional explanations. Human Resource Management Review, 21, 68-79. doi:10.1016/j.hrmr.2010.09.012

- Bruzzo, F., \& Basso, J. (2012). Article review measuring competing explanations of human resources management practices through the Cranet survey: cultural versus institutional explanations. Venezia.

- Budhwar, P. S., \& Debrah, Y. (2001). Rethinking comparative and cross-national human resource management research. The International Journal of Human Resource Management, 12(3), 497-515. Doi: 10.1080/713769629

- Budhwar, P. S., \& Sparrow, P. R. (2002). An integrative framework for understanding crossnational Human Resource Management practices. Human Resource Management Review, 12, 377 403.

- Chu, R. A., \& Wood Jr., T. (2008). Cultura organizacional brasileira pós-globalização: global ou local? Revista de Administração Pública, 42(5), 969-991.

- Coltro, A. (2009). Seção de pessoal, departamento de pessoal, administração de pessoal, administração de relações industriais, administração de Recursos Humanos, Gestão de Recursos Humanos, Gestão de Pessoas, ... ou o multiforme esforço do constante jogo. Revista de Administração Da UNIMEP, 7(1), 41-60.

- Cranet. (2011). Cranet survey on comparative Human Resource Management: international executive report 2011.

- Croucher, R., Brookes, M., Wood, G., \& Brewster, C. (2010). Context, strategy and financial participation: a comparative analysis. Human Relations, 63(6), 835-855. doi: 


\section{$10.1177 / 0018726709343654$}

- Croucher, R., Gooderham, P., \& Parry, E. (2006). The influences on direct communication in British and Danish firms: country, "Strategic HRM" or unionization? European Journal of Industrial Relations, 12(3), 267-286. doi: $10.1177 / 0959680106068913$

- Delery, J. E., \& Doty, D. H. (1996). Modes of theorizing in Strategic Human Resource Management: tests of universalistic, contingency, and configurational performance predictions. Academy of Management Journal, 39(4), 802-835. doi: $10.2307 / 256713$

- Demo, G., Fogaça, N., Nunes, I., Edrei, L., \& Francischeto, L. (2011). Políticas de Gestão de Pessoas no novo milênio: cenário dos estudos publicados nos períodos da área de administração entre 2000 e 2010. RAM. Revista de Administração Mackenzie, 12(5), 15-42.

- Fávero, L. P., Belfiore, P., Silva, F. L. da, \& Chan, B. L. (2009). Análise de dados: modelagem multivariada para tomada de decisões (1a. ed.). Rio de Janeiro: Editora Campus.

- Fischer, A. L. (2002). Um resgate conceitual e histórico dos modelos de gestão de pessoas. In As pessoas na organização (15th ed., pp. 11-34). São Paulo: Editora Gente.

- Gooderham, P., \& Nordhaug, O. (2010). One European model of HRM? Cranet empirical contributions. Human Resource Management Review, 21(1), 27-36. doi: 10.1016/j.hrmr.2010.09.009

- Gooderham, P., Nordhaug, O., \& Ringdal, K. (1999). Institutional and rational determinants of organizational practices: Human Resource Management in European firms. Administrative Science Quarterly, 44, 507-531.

- Gooderham, P., Parry, E., \& Ringdal, K. (2008). The impact of bundles of strategic human resource management practices on the performance of European firms. The International Journal of Human Resource Management, 19(11), 20412056.

- Guest, D. (1989). Personnel and HRM: can you tell the difference? Personnel Management Journal, 48-51.

- Gurkov, I., Zelenova, O., \& Saidov, Z. (2012). Mutation of HRM practices in Russia: an application of CRANET methodology. The International Journal of Human Resource Management, 23(7), 1289-1302. doi: 10.1080/09585192.2011.581633
- Hall, P. A., \& Soskice, D. (2001). Varieties of capitalism: the institutional foundations of comparative advantage. Oxford: Oxford University Press.

- Hofstede, G. (2001). Culture's consequences: comparing values, behaviors, institutions and organizations across nations (2 nd.). Thousand Oaks: SAGE Publications Ltd.

- Hofstede, G., Hofstede, G. J., \& Minkov, M. (2010). Cultures e organizational: software of the mind (3 rd.). New York: McGraw-Hill Education.

- Kramar, R. (2012). Trends in Australian Human Resource Management: what next? Asia Pacific Journal of Human Resources, 50(2), 133-150. doi: 10.1111/j.1744-7941.2011.00009.x

- Kramar, R., \& Parry, E. (2014). Strategic Human Resource management in the Asia Pacific region: similarities and differences? Asia Pacific Journal of Human Resources, 52(March), 400-419.

- Lacombe, B. M. B., \& Tonelli, M. J. (2001). O discurso e a prática: o que nos dizem os especialistas e o que nos mostram as práticas das empresas sobre os modelos de Gestão de Recursos Humanos. Revista de Administração Contemporânea, 5(2), 157-174.

- Larsen, H. H., \& Brewster, C. (2003). Line management responsibility for HRM: what is happening in Europe? Employee Relations, 25(3), 228-244. doi: 10.1108/01425450310475838

- Lazarova, M., Morley, M., \& Tyson, S. (2008). International comparative studies in HRM and performance - the Cranet data. The International Journal of Human Resource Management, 19(11), 1995-2003. doi: 10.1080/09585190802404239

- Lemos, A. H. da C., Santos, D. M., \& Dubeux, V. J. C. (2013). Práticas de gestão de pessoas individualistas ou coletivistas : o que brasileiros e norteamericanos demandam? Revista ADM.MADE, 17(1), 80-100.

- Lepak, D. P., \& Shaw, J. D. (2008). Strategic HRM in North America: looking to the future. The International Journal of Human Resource Management, 19(8), 1486-1499. doi: 10.1080/09585190802200272

- Malhotra, N. K. (2006). Pesquisa de marketing: uma orientação aplicada (4th ed.). Porto Alegre: Bookman.

- Martín-Alcázar, F., Romero-Fernández, P. M., \& Sánchez-Gardey, G. (2005). Strategic Human Resource Management: integrating the universalistic, contingent, configurational and 
contextual perspectives. International Journal of Human Resource Management, 15(5), 633-659. doi: 10.1080/09585190500082519

- Mayrhofer, W., Brewster, C., Morley, M. J., \& Ledolter, J. (2011). Hearing a different drummer? Convergence of Human Resource Management in Europe - a longitudinal analysis. Human Resource Management Review, 21, 50-67. doi: 10.1016/j.hrmr.2010.09.011

- Ministério do Trabalho e do Emprego. (2014). CAGED anuário RAIS. Brasília. Retrieved from http://bi.mte.gov.br/bgcaged/caged_anuario_rais /caged_anuario_raistela90.php

- Morley, M. J., \& Collings, D. G. (2004). Contemporary debates and new directions in HRM in MNCs: introduction. International Journal of Manpower, 25(6), 487-499. doi: 10.1108/01437720410560406

- Müller-Camen, M. (1999). Unitarism, pluralism, and Human Resource Management in Germany. Management International Review, 39(3), 125144.

- Paauwe, J., \& Boselie, P. (2005). HRM and performance: what' s Next? In CAHRS - Center for Advanced Human Resource Studies. Ithaca - NY: Cornell University ILR School.

- Parry, E., Dickmann, M., \& Morley, M. (2008). North American MNCs and their HR policies in liberal and co-ordinated market economies. The International Journal of Human Resource Management, 19(11), 2024-2040. doi: 10.1080/09585190802404262

- Peng, M. W. (2005). Perspectives-from China strategy to global strategy. Asia Pacific Journal of Management, 22(2), 123-141. doi: 10.1007/s10490-005-1251-3

- Piellusch, M., \& Taschner, G. B. (2009). Indicadores de $\mathrm{RH}$ no setor hoteleiro: um estudo nas maiores redes no Brasil. Organização \& Sociedade, 15(51), 665-686.

- Poutsma, E., Ligthart, P. E. M., \& Dietz, B. (2013). HRM policies and firm performance: the role of synergy of policies. In E. Parry, E. Stavrou, \& M. Lazarova (Eds.), Global Trends in Human Resource Management (1st ed., pp. 78-102). London: Palgrave Macmillan.

- Poutsma, E., Ligthart, P. E. M., \& Veersma, U. (2006). The diffusion of calculative and collaborative HRM practices in European firms. Industrial Relations, 45(4), 513-546. doi: 10.1111/j.1468-232X.2006.00442.x
- Rizov, M., \& Croucher, R. (2009). Human Resource Management and performance in European firms. Cambridge Journal of Economics, 33(1), 253-272. doi: 10.1093/cje/ben037

- Rousseau, D. M., \& Arthur, M. B. (1999). The boundaryless Human Resource function: building agency and community in the new economic era. Organizational Dynamics, 27(4), 7-18.

- Schuler, R. S. (1992). Strategic Human Resources Management: linking the people with the strategic needs of the business. Organizational Dynamics, 21(1), 18-32.

- Schuler, R. S., \& Jackson, S. E. (2005). A quartercentury review of Human Resource Management in the US: the growth in importance of the international perspective. Management Revue, 16(1), 11-35.

- Silva, S. R., \& Azzuz, E. (2003). Gestão de Pessoas na indústria de calçados de Franca: departamento burocrático ou parceiro estratégico? Revista Eletrônica de Administração - FACEF, 2(3), 1-20.

- Stavrou, E., \& Kilaniotis, C. (2010). Flexible work and turnover: an empirical investigation across cultures. British Journal of Management, 21(2), 541-554. doi: 10.1111/j.1467-8551.2009.00659.x

- Subramony, M. (2006). Why Organizations Adopt some Human Resource Management Practices and Reject Others: An Exploration of Rationales. Human Resource Management, 45(2), 195-210. doi: 10.1002/hrm.20104

- Supangco, V. T. (2012). Strategic HR practices in some organizations in the Philippines. Philippine Management Review, 19, 35-48.

- Tanure, B., Evans, P., \& Cançado, V. L. (2010a). As Quatro Faces de RH : Analisando a Performance da Gestão de Recursos Humanos em Empresas no Brasil. Revista de Administração Contemporânea RAC, 14(2), 594-614.

- Tanure, B., Evans, P., \& Cançado, V. L. (2010b). As Quatro Faces de RH : Analisando a Performance da Gestão de Recursos Humanos em Empresas no Brasil. Human Resource Management, 14(2), 594614.

- Tanure, B., Evans, P., \& Pucik, V. (2007). A Gestão de Pessoas no Brasil: virtudes e pecados capitais (1st. ed.). Rio de Janeiro: Elsevier.

- Tinoco, J. E. P. (2005). Gestão estratégica do capital humano. Organizações em Contexto, 1(1), 13-70.

- Tonelli, M. J., Caldas, M., Lacombe, B. M. B., \& Tinoco, T. (2003). Produção acadêmica em 
Recursos Humanos no Brasil: 1991-2000. Revista de Administração de Empresas, 43(1), 1-18.

- Uysal, G. (2014). Convergence or divergence between European HRM. Journal of Business and Economics, 5(10), 1923-1928. doi: 10.17265/2328-7144/2016.02.004

- Vasconcelos, I., Mascarenhas, A. O., \&
Vasconcelos, F. (2004). Paradoxos organizacionais, Gestão de Pessoas e tecnologia na Souza Cruz. RAE Eletrônica, 3(2), 1-20.

- Whitley, R. (1999). Divergent capitalisms: the social structuring and change of business systems. Oxford: Oxford University Press.

\section{SOBRE OS AUTORES:}

- Tatiani dos Santos Zuppani é .Doutora em Administração pela Universidade de São Paulo. Pesquisadora na área de Gestão de Recursos Humanos e Carreira.E-mail: tszuppani@gmail.com

- André Luiz Fischer é Professor no Departamento de Administração da FEA USP ministrando aulas e orientando trabalhos de pesquisa, mestrado doutorado e pós-doutorado. Coordena cursos de especialização em Gestão de Recursos Humanos atuando como consultor e pesquisador na Faculdade FIA de Administração e Negócios. E-mail: afisher@usp.br 


\section{Comparative international management of human resources and human resources management in Brazil: An analysis in view of the calculative and collaborative models}

Tatiani dos Santos Zuppani and André Luiz Fischer

University of São Paulo - USP, São Paulo, SP, Brazil.

\section{ARTICLE DETAILS}

\section{Article history:}

Received 11 January 2016

Accepted 28 April 2016

Available online in 31 April 2016

Double Blind Review System

\section{Scientific Editor}

Eduardo Eugênio Spers

\section{Keywords:}

Comparative International Management of Human Resources; Strategic Human

Resources Management;

Calculative Model;

Collaborative Model

\begin{abstract}
The aim of this study was to analyze the adoption of calculative and collaborative practices dominating comparative international human resources management, according to the different profiles of the areas of Human Resources Management (HRM) of private organizations operating in Brazil. The method employed was a Survey, operated by means of an electronic questionnaire on HRM practices and organizational characteristics. A total of 326 respondents was obtained. Initially a cluster was conducted, in which respondents were clustered into four groups with different HRM profiles. The use of calculative and collaborative practices was compared in the four groups formed through the ANOVA (analysis of variance) collection of statistical models. The main findings showed that the strategic group was the one with the highest average of adoption of calculative and collaborative practices. The Communicative HRM group showed a higher propensity to collaborative practices and the Formalized HRM group would adopt calculative practices, although none of the groups showed an average of adoption than the Strategic HRM group. This suggests that it is necessary to learn how to deal with different aspects of the management of people in organizations operating in Brazil.
\end{abstract}

(C) 2016 Internext | ESPM. All rights reserved!

To cite this article:

Zuppani, T. S. \& A. L. Fischer (2016) Gestão internacional comparativa de recurso humanos e gestão de recursos humanos no Brasil: Uma análise frente aos modelos calculativos e colaborativos. Internext - Revista Eletrônica de Negócios Internacionais, 11 (2), p. 49-65.

To link to this article: $h t t p: / / d x . d o i . o r g / 10.18568 / 1980-4865.11249-65$ 